\title{
Long Term Outcome of Preoperative Isolated Limb Infusion and External Irradiation in Management of Locally Advanced Extremity Soft Tissue Sarcoma
}

\author{
Mohamed A. Hegazy ${ }^{*}, 1$, Waleed Elnahas ${ }^{1}$, Omar Farouk ${ }^{1}$, Mahmoud Mosbah ${ }^{1}$, Mohamed Hafez ${ }^{1}$, \\ Sherif Kotb ${ }^{1}$, Hanem Sakr ${ }^{2}$, Waleed Abo Zeid ${ }^{2}$, Sayed Hendawy ${ }^{2}$, Talal Amer ${ }^{3}$ and Rifaat Hegazi ${ }^{4}$
}

Departments of ${ }^{1}$ Surgical Oncology, ${ }^{2}$ Clinical Oncology and Radiotherapy, and ${ }^{3}$ Diagnostic Radiology, Faculty of Medicine, Mansoura University, Egypt; ${ }^{4}$ Pittsburgh School of Medicine, USA

\begin{abstract}
Background: We report our long term results of isolated limb infusion (ILI) in cases of locally advanced softtissue sarcoma (ASTS) of the extremities.

Methods: Forty cases of ASTS received ILI with doxorubicin. Preoperative external beam radiotherapy started within 3-7 days after ILI was administered. After 3-7 weeks, surgery was performed aiming at limb preservation. The long term outcome of these cases (Group I) was reported and compared to the outcome of comparable group of patients followed prospectively and treated by neoadjuvant systemic chemotherapy (NACT) and external irradiation (Group II).

Results: The study included 40 cases in Group I, and 46 cases in Group II. Overall response to preoperative treatment was $85 \%$ in Group I versus $43 \%$ in Group II. Wide local excision was performed for $75 \%$ of Group I patients and in $34 \%$ of Group II cases. After a median follow up period of 76 months, local recurrence rate was 35\% in Group I and $67 \%$ in Group II $(\mathrm{P}=0.02)$. The overall survival rate was $60 \%$ in Group I and $35 \%$ in Group II $(\mathrm{p}=0.008)$. Only initial response to ILI was associated with overall survival in Group I.
\end{abstract}

Conclusion: The outcome of ILI in management of ASTS is significantly better than systemic NACT in terms of disease free and overall survival.

Keywords: Isolated limb infusion, extremity soft tissue sarcoma, long term survival.

\section{INTRODUCTION}

The management of locally advanced extremity soft tissue sarcomas continues to be a challenge. The prognosis of patients with advanced soft-tissue sarcoma (ASTS) remains poor for decades with a median survival of at best 12 months [1]. Current improvement in patients' quality of life and survival in STS have resulted from contribution of many advances in its management. There have been contributions from centralization of service, multidisciplinary management, improved surgical technique, multimodality therapy and the development of limb sparing surgery. However, controversies surround the main pillars of treatment such as the type of surgery (e.g. indications for limb salvage) and the neo-adjuvant and adjuvant treatment modalities, such as radiation therapy or chemotherapy [2].

Neoadjuvant chemotherapy (NACT) in treatment of patients with STS is an area of controversies where small progress has been made over the past years. Sarcomas show variability in response to chemotherapy, which emphasizes the heterogeneity of this tumor entity. It is probably that the lack of distinction of sarcoma subtypes with regard to cytostatic therapies might be responsible for the conflicting

*Address correspondence to this author at the Oncology Center, Mansoura University, Egypt; Tel: +20-01222447066; Fax: +20-0502358023;

E-mail: mhegazy68@yahoo.com data we have when patients are treated in a neoadjuvant setting [3].

Data on prognostic and predictive factors for outcome to neoadjuvant chemotherapy are not available from large series. Identification of such factors is essential for patient management and clinical trial design, in particular for STS given its heterogeneity. While tumor size and grade are wellestablished risk factors for local or systemic recurrence, other factors (e.g. tumor location, histologic type, margin status) have only been vaguely defined, which complicates the development of evidence-based treatment algorithms [4].

Neoadjuvant isolated regional chemotherapy is an attractive treatment option because it allows much larger doses of chemotherapy to be delivered to the tumor with minimal systemic toxicity. Furthermore, where the tumor responds to the therapy, vital anatomical structures may be able to be preserved at the time of subsequent surgical excision, and previously unresectable tumors may be converted into resectable ones [5]. Isolated limb infusion (ILI) is a minimally invasive technique of delivering regional chemotherapy in ASTS patients. This technique was developed and implemented in the early 1990s by Thompson et al., at Sydney melanoma unit with the objective of obtaining the benefit of conventional isolated limb perfusion (ILP) without incurring its major disadvantages [6]. 
The authors present their initial results of isolated limb infusion (ILI) in patients with ASTS of the extremities in 2006 [7]. The goal of the present study is to discuss the results of ILI on ASTS cases on the long term (more than 5 years). We compared the survival of ILI cases with a comparable group of patients that received neoadjuvant systemic chemotherapy and external irradiation followed by limb salvage surgery. In addition, analysis for prognostic factors was performed aiming to establish independent predictive factors for best overall response (RR), disease free survival (DFS) and overall survival (OS).

\section{PATIENTS AND METHODS}

\section{Patients}

The data of the 40 cases that underwent ILI (Group I) since 2005 was collected and compared to a comparable group of cases that received NACT and external irradiation (Group II). Therefore, we retrospectively reviewed the medical records of 234 extremity STS patients treated at our institutions from 1998 to 2004. Among those cases, 153 cases were locally advanced. After applying the inclusion criteria, excluding patients that lost follow up $(n=23)$ and matching the patients in both groups regarding age, sex, tumor site, size, type, we got a cohort of 46 patients that could be compared as a control group. Inclusion criteria were the following: 1- high grade sarcoma, 2- large size $>5 \mathrm{~cm}, 3-$ proximity to a neurovascular bundle and/or the bone, and 4a minimum of 5 years follow up duration since the initiation of treatment. Patients with the chemosensitive subtypes Ewing sarcoma, rhabdomyosarcoma, and desmoplastic small round cell tumor were excluded from the study.

This study was performed retrospectively from our data base. However, patients treated with ILI since 2005 gave written consents that their follow up data will be used in this research and our IRB approved this.

\section{Treatment Programs}

1. Preoperative isolated limb infusion (ILI) and external irradiation: ILI was performed using the same technique as had been described earlier by Thompson et al., [6], and the investigators [7]. In brief, standard radiologic catheters were inserted percutaneously into the axial artery and vein of the disease-bearing limb via the contralateral groin. The catheter tips were positioned at the level of the major feeding vessels of the tumor. Then the contrast medium (urovidio) was injected through the catheter to evaluate the vasculature of the tumor region and to obtain angiographic run, determining the feeding vessel of the tumor (Fig. 1). When it was confirmed that the position of the angiographic catheter was satisfactory, a pneumatic tourniquet was inflated around the root of the limb to be treated and the cytotoxic agent (doxorubicin 0.7 and $1.4 \mathrm{mg} / \mathrm{kg}$ for the upper and the lower limbs, respectively) was infused into the isolated limb via the arterial catheter. For the duration of the ILI procedures (15-25 $\mathrm{min})$, the infusate was then continually circulated by repeated aspiration from the venous catheter and reinjection into the arterial catheter by using a syringe attached to a threeway tap in the circuit. After 15-25 min the limb was flushed with $1 \mathrm{~L}$ of Hartman's solution via the arterial catheter. The limb tourniquet was then deflated to restore normal limb circulation and the catheters were removed. External beam radiotherapy (35 Gy in ten fractions) started within 3-7 days after ILI. After 3-7 weeks, limb sparing surgery was performed. Usually this period allowed for enough shrinkage of the tumor and allowed the soft tissues to recover from the inflammatory reaction of radiotherapy.

2. Neoadjuvant systemic chemotherapy (NACT) and external irradiation: The systemic chemotherapy of each cycle consisted of doxorubicin (adriamycin) 50 $\mathrm{mg} / \mathrm{m} 2$ on day 1 , etoposide $125 \mathrm{mg} / \mathrm{m} 2$ on days 1 and 4 , and ifosfamide $1250 \mathrm{mg} / \mathrm{m} 2$ for $60 \mathrm{~min}$ on days 1 4. A total of 3-4 neoadjuvant courses were given before assessment of the tumor response. External beam radiotherapy (35 Gy in ten fractions) started within 3-7 days after completion of the chemotherapy cycles. All cases were referred to surgery after finishing their neoadjuvant protocols.

\section{Treatment Evaluation and Statistics}

After neoadjuvant protocols, systemic and limb toxicity and tumor response were assessed regularly. Systemic toxicities were graded according to the World Health Organization (WHO) grading scale. The scale proposed by Wieberdink et al., [8] was used to assess limb toxicity. After 3 - 6 weeks, the tumor response was evaluated. Clinical response was classified as follow: complete response (CR: complete disappearance of all measurable or evaluable tumor for a minimum of 4 weeks), partial response (PR, greater than $50 \%$ reduction of the tumor volume lasting at least 4 weeks), minor response (MR, reduction of the tumor volume by less than $50 \%$ for at least 4 weeks), stable disease (SD, less than $25 \%$ increase in the tumor volume for at least 4 weeks), and progressive disease (PD, an increase of greater than or equal to $25 \%$ of the tumor volume and/or occurrence of new lesion). In this study, response to chemotherapy was analyzed as a binary variable: responders were those who were reported as having achieved a complete, partial, or minimal radiologic response (according to the WHO criteria); all other patients were classified as non-responders.

By definition, patients were classes as NED (no evidence of disease) at the time of surgery in cases of R0 (negative margins on frozen section examination), R1 (positive margins on frozen section). Patients with R2 (residual disease), were considered non- NED.

Overall survivals (OS) were measured from the start of treatment until death from any cause or last follow up. Disease free survival (DFS) was defined as the time from start of treatment until radiologic documentation of disease recurrence or last follows up. For the patients not followed up in our institutions or missed a follow up visit, the referring surgeon or the patient's family was contacted to determine the patient's long term follow up status.

Survival curves were calculated by the Kaplan Meier method. Comparison of survival curves were performed using the log rank test (Mantel-Cox). Age, sex, histologic type, grade, site of the tumor, type of surgery and margin status were investigated as potential prognostic factors for OS and DFS by means of a univariate log rank analysis. The 
A
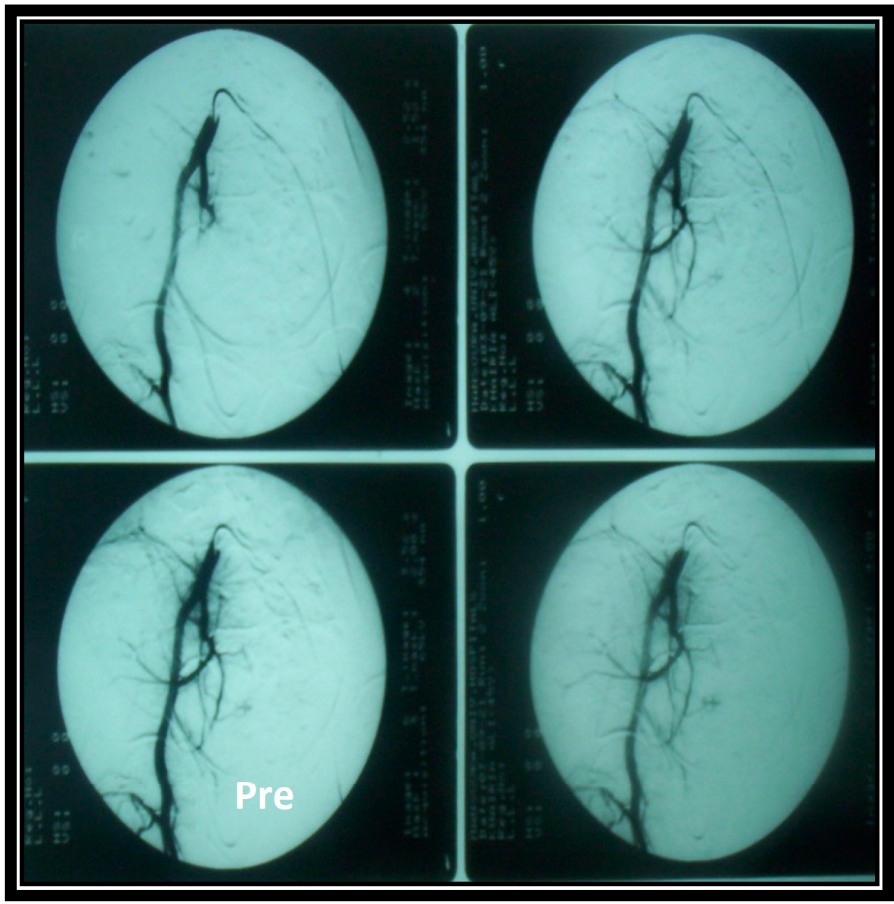

B

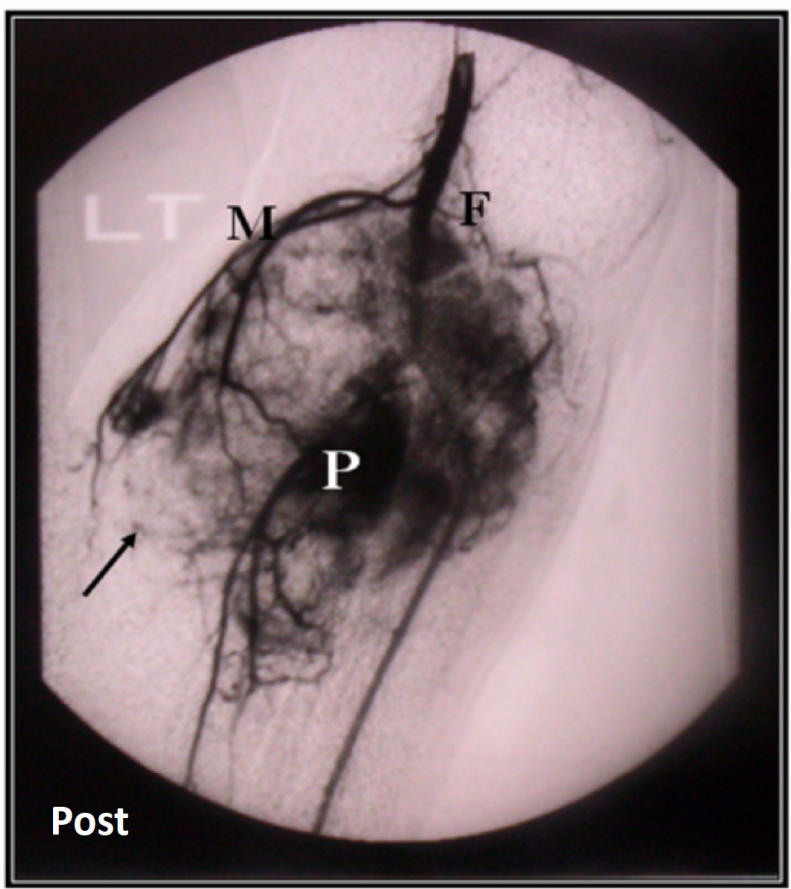

Fig (1). A. ILI; the angiographic catheter was introduced over the guide wire for enough distance upward into the aorta till reaching bifurcation of the aorta. B. angiogram showing a hypertrophied feeding artery (F), marginal pathologic vessels (M), tumor plush (P), and intratumoral microaneurysms (arrow).

independent significance of variables was assessed in a multivariate Cox regression analysis.

\section{RESULTS}

\section{Patients' Characteristics}

We retrospectively reviewed the medical records of 234 extremity STS patients treated at our institutions from 1998 to 2004. Among them, 153 cases were locally advanced and 97 cases received NACT and preoperative irradiation. After applying the inclusion criteria and matching the 2 groups regarding age, sex, tumor site, size and type, a total of 46 cases were picked as a control group (Group II). These patients were compared to 40 patients who were treated by preoperative ILI and external irradiation (Group I). The mean ages $( \pm \mathrm{SD})$ of the two groups at the time of diagnosis were $42( \pm 13)$. Men constituted $71 \%$ of the patient population $(n=65)$. The tumor site was upper extremity (at or beyond the shoulder) in $22 \%$ of the patients $(n=19)$ and lower extremity (at or beyond the groin in $78 \%$ of patients $(n=67)$. Tumor size was defined according to the pathologic report as the maximum diameter of the tumor. Tumors $>$ $5 \mathrm{~cm}$ were present in $95 \%$ of the patients. Of the 86 patients, $42 \%(n=36)$ had low grade tumors and $58 \%(n=50)$ were high grade (Table 1).

\section{Response and Surgery Results}

In Group I; disease control rate was $85 \%$ ( $n=34$ patients). Twelve cases (30\%) showed partial response and $22(55 \%)$ showed minimal response. All patients showed an increase
Table 1. Patients Characteristics of the 3 Groups

\begin{tabular}{|c|c|c|c|}
\hline & $\begin{array}{c}\text { Group I } \\
\text { ILI } \\
(n=40)\end{array}$ & $\begin{array}{c}\text { Group II } \\
\text { Systemic CT } \\
(n=46)\end{array}$ & P Value \\
\hline Age $($ mean \pm SD $)$ & $42 \pm 10.3$ & $41 \pm 14$ & 0.95 \\
\hline \multicolumn{4}{|l|}{ Sex } \\
\hline Male & 33 & 38 & 0.33 \\
\hline Female & 7 & 8 & \\
\hline \multicolumn{4}{|l|}{ Tumor Size $(\mathrm{cm})$} \\
\hline Median & 11.7 & 11.3 & 0.24 \\
\hline range & $5-14$ & $7-13$ & \\
\hline \multicolumn{4}{|l|}{ Tumor site } \\
\hline Lower limb & 35 & 39 & 0.31 \\
\hline Upper limb & 5 & 7 & \\
\hline \multicolumn{4}{|l|}{ Pathology } \\
\hline MFH & 16 & 13 & 0.12 \\
\hline Liposarcoma & 18 & 21 & \\
\hline Fibrosarcoma & 12 & 2 & \\
\hline Spindle cell sarcoma & 0 & 2 & \\
\hline Synovial sarcoma & 0 & 3 & \\
\hline Neurogenic sarcoma & 4 & 5 & \\
\hline \multicolumn{4}{|l|}{ Tumor Grade } \\
\hline Low & 22 & 25 & 0.46 \\
\hline High & 18 & 21 & \\
\hline
\end{tabular}


in the extent of necrosis and extensive cystic degeneration of their tumors as detected in MRI. In Group II, disease control rate of neoadjuvant chemotherapy was $43 \% \quad(n=20)$. Radiographic responses consisted of seven patients (15\%) with partial response and 13 cases (28\%) with minimal response. There was no complete response in either group.

Surgical resection was carried out for 36 cases (34 responders and 2 cases that showed stable disease). Local excision (wide or marginal) was done in 30 cases $(75 \%)$ and compartmental excision was needed for 6 cases only $(15 \%)$. Four cases showed progressive disease; amputation was performed in three cases and the fourth case refused amputation and was referred for EBRT (external beam radiotherapy) and/or systemic chemotherapy. For cases who underwent limb salvage surgery $(n=36)$, R0 resection was achieved in 29 cases $(72.5 \%)$ and R1 in 7 cases $(17.5 \%)$. Only one case in this group needed reconstructive surgical procedure (arterial graft).

In Group II, 20 cases showed response and 17 patients showed stable diseases. These 37 cases underwent surgical excision of their primary tumors. Local excision (wide or marginal) was done in 20 cases (43\%), compartmental excision in 15 cases $(33 \%)$, and debulking in 2 case $(4 \%)$. $\mathrm{R} 0$ resection could be performed in 18 cases $(39 \%), \mathrm{R} 1$ resection was achieved in 17 cases $(37 \%)$, and R2 in the 2 debulking cases (4\%). Seven cases in Group II needed reconstructive procedures ( 3 arterial grafts, 1 nerve graft, and 3 pedicled fascio-cutaneous flaps). Nine cases showed progressive disease on systemic neoadjuvant systemic chemotherapy. Amputation was performed in 6 cases, and the other 3 cases developed systemic metastasis. The surgical outcome of the study groups are summarized in Table 2.

Table 2. Surgical Outcome of the Study Groups

\begin{tabular}{|c|c|c|c|}
\hline & $\begin{array}{c}\text { Group I } \\
\text { ILI } \\
(n=40)\end{array}$ & $\begin{array}{c}\text { Group II } \\
\text { Systemic CT } \\
(n=46)\end{array}$ & P Value \\
\hline \multicolumn{4}{|l|}{ Surgery performed: } \\
\hline local excision & $30(78.5 \%)$ & $20(43 \%)$ & 0.025 \\
\hline Compartmental excision & $6(15 \%)$ & $15(33 \%)$ & \\
\hline Debulking & 0 & $2(4 \%)$ & \\
\hline Amputation & $3(7.5 \%)$ & $6(13 \%)$ & \\
\hline None & $1(2.5 \%)$ & $3(7 \%)$ & \\
\hline \multicolumn{4}{|l|}{$\begin{array}{l}\text { Resection status in limb } \\
\text { sparing surgeries: }\end{array}$} \\
\hline R0 & $29(72.5 \%)$ & $18(39 \%)$ & 0.037 \\
\hline $\mathrm{R} 1$ & $7(17.5 \%)$ & $17(37 \%)$ & \\
\hline $\mathrm{R} 2$ & $0(0 \%)$ & $2(4 \%)$ & \\
\hline \multicolumn{4}{|l|}{ Reconstruction } \\
\hline Yes & $1(2.5 \%)$ & $7(15 \%)$ & 0.014 \\
\hline No & $39(97.5 \% \%)$ & $39(85 \%)$ & \\
\hline
\end{tabular}

Extensive histopathological examination for response after surgery was performed in all specimens. Histologic response was significantly better $(\mathrm{P}=0.002)$ in ILI cases (32 cases, $80 \%$ ), than in Group II cases (14 cases, 30\%).

In group I, 34 cases completed adjuvant chemotherapy, and 6 cases didn't receive any postoperative treatment. In Group II, 42 cases completed adjuvant treatment.

\section{Treatment Related Toxicity}

During neoadjuvant and adjuvant treatment, nonhaematological toxicity was usually mild (WHO grade $1)$; severe side effects were not seen in our study population. The most frequent side effects were alopecia, which was observed for all patients, and nausea, which was seen in $36 \%$ of patients. Hematological toxicity mainly consisted of leucopenia and lesser extent thrombocytiopenia. In Group II cases, 44 patients $(96 \%)$ experienced leucopenia, commonly WHO grade $3(n=26,57 \%)$, and grade $4(n=10,22 \%)$. The rate of systemic complications was significantly lower in Group I cases $(\mathrm{P}=0.002)$ where only 12 cases $(30 \%)$ developed leucopenia mainly WHO grade 2 .

Local morbidity to ILI developed in 12 patients $(30 \%)$. These were graded using the scale proposed by Wieberdink et al., [8]. Eight cases experienced grade 2 complications (slight edema and/or erythema) and four cases experienced grade 3 complications with only one case having slight motility impairment. No long term systemic or local toxicity was encountered in the two groups.

\section{Relapse and Survival}

After a median follow up period of 76 months (range: 13114 months), ILI cases showed 35\% local recurrence rate with a median time of 29 months (range: 11-98). This result was significantly better $(\mathrm{P}=0.02)$ than Group II cases that showed a local recurrence rate of $67 \%$ with a median time of 13 months (range 9- 102). (Fig. 2)

\section{Survival Functions}

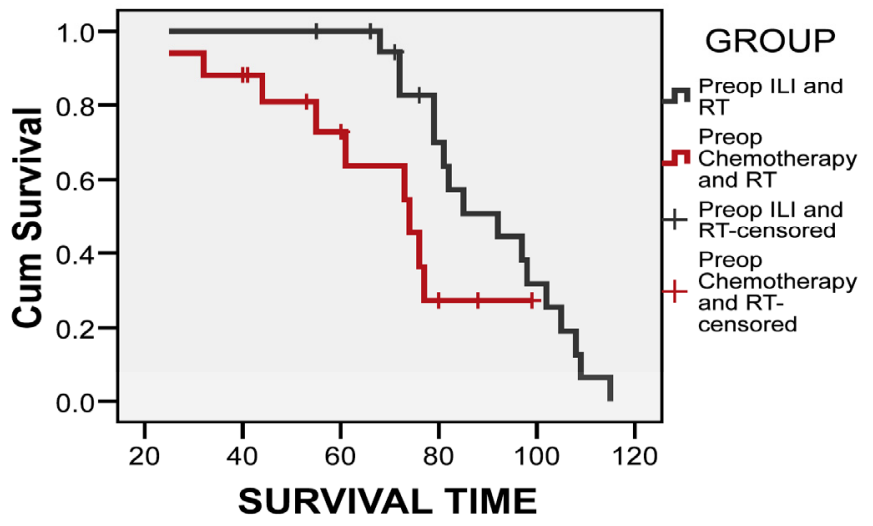

Fig. (2). Kaplan Meir estimate of disease free survival in the studied groups.

Univariate analysis of DFS in the whole cohort of patients revealed significantly better survival with cases treated surgically by wide local excision $(\mathrm{P}=0.032)$, and non metastatic cases $(P=0>002)$. In each group there was no significant difference in local recurrence rates according to age, sex, site of the tumor, size, stage, histologic type, grade, type of surgery or margin status. Only in group I, local recurrence rate was significantly affected by the tumor size after ILI $(\mathrm{P}=0.004)$ 
Distant metastasis occurred in $40 \%$ of group I cases (median time 48 months, range: 14- 104), and in $63 \%$ in group II (median time 42 months, range: 7- 96). The difference just failed to show statistical significance $(\mathrm{P}=0.07)$. Margin status only was shown by logistic regression to be a prognostic factor related to metastasis in the whole population of patients $(\mathrm{P}=0.002)$. Metastasis was not influenced by any of the study parameters in each group.

At present, $60 \%$ of our ILI patients are alive with a median survival of 105 months (range 22-114). This result was significantly better $(\mathrm{P}=0.03)$ than Group II cases $\{35 \%$ alive with a median of 82 months (range: 13-96)\} (Fig. 3). In the whole population of patients, overall survival was significantly correlated with tumor size after treatment $(\mathrm{P}=0.001)$, type of surgery performed (WLE versus compartmental; $\mathrm{P}=0.003)$ (Fig. 4), margin status $(\mathrm{P}=0.0059)$, presence of local recurrence $(\mathrm{P}=0.0007)$, and presence of metastasis $(\mathrm{P}=<0.0001)$. In group I overall survival was correlated with the tumor size after ILI $(\mathrm{P}=0.0005)$, and surgical margin $(\mathrm{P}=0.04)$. In group II, OS was correlated only with type of surgery $(\mathrm{P}=0.04)$. Using a multivariate analysis with logistic regression, parameters (excluding response to chemotherapy) independently correlated to a poor 5-year survival rate were similar to those previously identified as independent prognostic variables for overall survival in the same series: tumor size after treatment (Relative Risk RR: 2.1), presence of local recurrence (RR: 2.3), distant metastases (RR: 4.5) and type of surgery performed (RR: 2.7).

\section{Survival Functions}

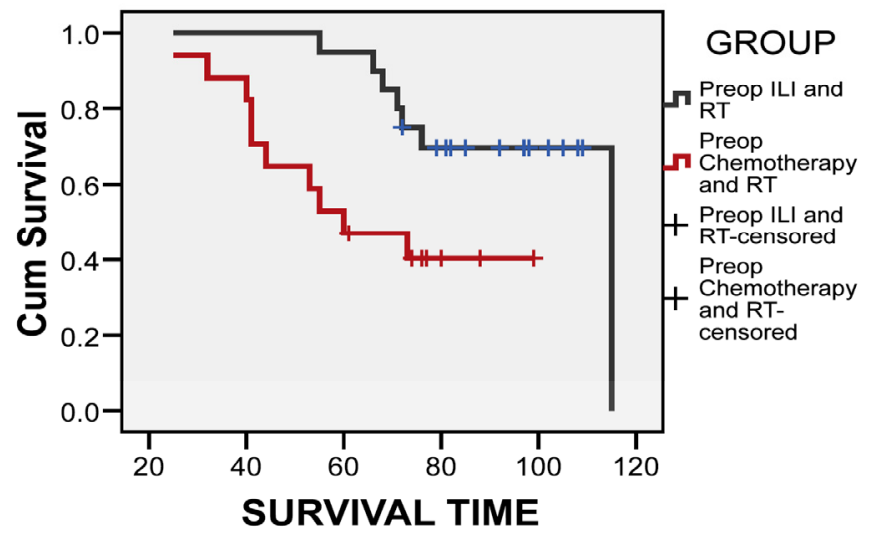

Fig. (3). Kaplan Meir estimate of overall survival in the studied groups.

The disease free and overall survival events in the study groups are summarized in Table $\mathbf{3}$.

\section{DISCUSSION}

Regional chemotherapy is an attractive treatment option for patients with advanced extremity sarcoma. It has been traditionally carried out using a procedure called isolated limb perfusion (ILP), first developed in New Orleans in the mid-1950s [9]. The technique was based on the proposal that vascular isolation and perfusion of the extremity with chemotherapy would allow regional drug concentrations several orders of magnitude higher than could be attained with systemic administration. ILP is technically complex, however, and associated with significant morbidity and cost.
Vascular isolation of the extremity for ILP requires surgical exposure and open cannulation of the artery and vein to the extremity. The extremity is then placed on bypass, requiring the presence of a perfusion team and a cardiopulmonary bypass machine in addition to surgical and anesthesia staff. ILI was developed at the Sydney Melanoma Unit (SMU) by Thompson and colleagues [6] as a simple alternative to ILP. Percutaneously placed catheters replace open surgical cannulation and the chemotherapy is recirculated manually so that no pump oxygenator is needed. Operating room time is approximately $1 \mathrm{~h}$, compared with the 4 or $5 \mathrm{~h}$ needed to perform ILP. Most importantly, Thompson and colleagues reported that ILI has an efficacy similar to ILP. They reported significant clinical responses in 135 patients treated with ILI, with $41 \%$ CR and 44\% PR [10].

\section{Survival Functions}

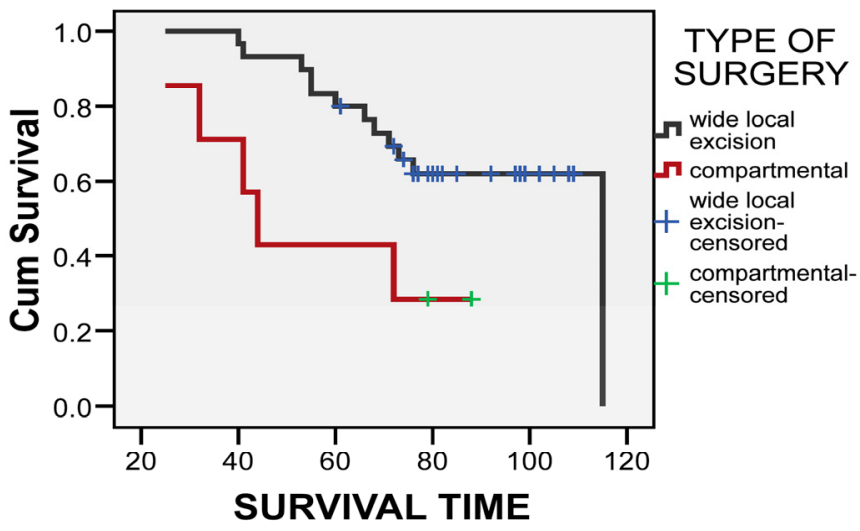

Fig. (4). Correlation between the type of surgery and overall survival in the whole patients' population.

Table 3. Disease Free and Overall Survival Events in the Study Groups

\begin{tabular}{|l|c|c|c|}
\hline & $\begin{array}{c}\text { Group I } \\
\text { ILI } \\
(\mathbf{n}=\mathbf{4 0})\end{array}$ & $\begin{array}{c}\text { Group II } \\
\text { Systemic CT } \\
(\mathbf{n}=\mathbf{4 6})\end{array}$ & P Value \\
\hline \hline $\begin{array}{l}\text { Local recurrence } \\
\text { Number (\%) }\end{array}$ & $14(35 \%)$ & $31(67 \%)$ & 0.02 \\
Treatment & 6 & 6 & \\
$\quad$ Re-excision & 4 & 2 & \\
CRT & & & \\
Metastasis & $16(40 \%)$ & $29(63 \%)$ & 0.07 \\
Number (\%) & 10 & 17 & \\
Site & 4 & 7 & \\
Lung & 2 & 5 & \\
Skeletal & & & \\
Brain & & & \\
& $24(60 \%)$ & $16(35 \%)$ & 0.03 \\
Overall survival & $16(40 \%)$ & $30(65 \%)$ & \\
Alive & 105 & 82 & \\
Dead & & & \\
Median survival (m) & & & \\
\hline
\end{tabular}

The authors report their experience with ILI in a cohort of 40 ASTS patients and showed that they can effectively be 
treated when a combination of pre-operative ILI and radiotherapy is administered. All patients underwent surgical resection 3-7 weeks after the pre-operative treatment [7]. Survival rates were not mentioned in this study because we were convinced that the follow up period was too short to draw conclusions. After a median follow up of 76 months (range: 13-114 months), local recurrence occurred in 35\% of cases with a median time of 29 months (range 11-98 months). Distant metastasis occurred in $40 \%$ of patients with a median time of 52 months. At present, $60 \%$ of ILI cases are still alive (Fig. 5) with a median survival of 105 months (range: 22-114 months). In a Sidney Melanoma Unit based study, the effect of ILI on STS was analyzed in 21 patients. Fourteen (67\%) patients underwent ILI as neo-adjuvant therapy prior to surgery and seven patients underwent ILI to treat inoperable recurrences or for palliation. $57 \%$ of the patients had a CR and limb salvage was achieved in $76 \%$. Local recurrence rate was $42 \%$ with a median recurrence free survival of 25 months. The overall disease specific survival was $62 \%$. A lower stage of disease was a significantly associated with longer survival ( $\mathrm{P}=0.008)$ [11].

A group from Switzerland reported their experience with ILP with TNF and melphalan for non resectable STS. After a mean follow up period of 38.9 months (4-159), 44\% of their patients survived more than 5 years. Local recurrence occurred in $37 \%$ of cases and they explained this high rate on one hand by a longer follow up time (more than 3 years) allowing the recurrence registration and on the other hand by a higher proportion of high grade and advanced stage sarcomas. Systemic metastasis occurred in $68 \%$ of cases [12]. In 2003, Noorda et al., reported 48\% 5- years disease free survival, but with $20 \%$ grade I and $41 \%$ stage I and II tumors [13]. Other series did not mention 5- years survival rates because of a shorter follow up.

In order to document a survival advantage of ILI, we compared our results with a comparable group of ASTS cases that was treated with neoadjuvant systemic chemotherapy.

In the literature, conflicting data have been presented concerning the role of NACT in treatment of high risk extremity sarcomas. Merice et al., [14], reported that only $12 \%$ of patients responded to chemotherapy enough to facilitate or simplify their tumor resection. Additionally $9 \%$ of patients required a larger surgery because of tumor progression despite of ongoing chemotherapy treatment. Menendez et al., [15], in a retrospective review, determined that there was no statistical significance in recurrence free survival or overall survival in a group of 82 patients who received three to four cycles of neoadjuvant doxorubicin, ifosfamide and cisplatin. In contrast, Eilber et al., [16] suggested that although the percentage of patients who respond is low, clinical benefit may be seen in a subset of patients. In the small group of patients (14\%) that demonstrated a complete response to chemotherapy ( $95 \%$ necrosis), the 10-year local recurrence rate in that group was $11 \%$ as compared to $23 \%$, and the 10 -year survival rate was $71 \%$ as compared to $55 \%$. The current clinical dilemma is that it is impossible to determine which patients will respond to chemotherapy, and unfortunately the percentage of patients that respond to treatment is near equivalent to the number of patients who progress on therapy and require larger resections [17]. In the only randomized clinical trial evaluating the effect of neoadjuvant chemotherapy upon disease-free and overall survival (EORTC 62874), 134 highrisk patients (with tumors of any grade $>8 \mathrm{~cm}$, grade II/III tumors $<8 \mathrm{~cm}$, or tumors either recurrent or residual from a prior operation) received three cycles of neoadjuvant AI (doxorubicin: $50 \mathrm{mg} / \mathrm{m} 2 /$ cycle as an intravenous bolus; ifosfamide: $5 \mathrm{~g} / \mathrm{m} 2 /$ cycle as a $24-\mathrm{hr}$ infusion) or surgery alone. The 5-year disease-free survival rate in the chemotherapy arm $(56 \%)$ was slightly higher than in the control arm (52\%) but was not powered for statistical significance [18]. In the present study, the results of systemic NACT are compatible with the previous negative reports. Overall response to treatment was present in $43 \%$ of cases. Although limb salvage surgery could be performed in most cases (35 cases, 74\%), NACT did not facilitate a wider resection; R1 resection rate was similar to R0 resection $(47 \%)$. Moreover the only R2 resection in the whole series of patients was among this group. Local recurrence rate was $67 \%$, distant metastasis occurred in $53 \%$ of cases, and $35 \%$ of them are still alive with a median survival of 82 months.

Age, sex, tumor size, grade and localization are declared as prognostic factors on overall survival in the literature [19]. Positive margin, age and localization of recurrences are the prognostic factors for local recurrence [20]. According to this study, the statistically significant prognostic factors influencing the overall survival were: size of tumor after neoadjuvant treatment (reflecting tumor response), type of surgery performed (WLE versus compartmental excision), margin status, presence of local recurrence, and presence of metastasis. Age, sex and tumor location were found to have no impact on overall survival. The factors influencing local recurrence were the type of surgery and presence of metastasis. In Group I cases, the only factor that had a significant impact upon both overall survival and disease free survival was the tumor size after ILI reflecting tumor response. This is consistent with the results of ILP in treating melanoma, where $\mathrm{CR}$ was a statistically significant positive prognostic indicator after long term follow up which may reflect a more favorable tumor biology [21]. Moncreiff et al., [11] reported that there was a trend for a CR to be associated with improved OS in their series of ASTS cases treated by ILI, but this just fails to reach statistical difference $(\mathrm{P}=0.07)$. Meanwhile radiologic tumor response did not affect local recurrence or overall survival. This is consistent with Issels et al., [22] who treated 59 ASTS patients with neoadjuvant 4 EIA (etoposide, ifosfamide, and doxorubicin) cycles combined with regional hyperthermia to be followed by surgery and adjuvant treatment. The authors stated that responding patients appeared to have survival rates that were similar to those patients who did not respond to preoperative systemic chemotherapy. In the whole cohort of these patients complaining locally advanced STS, cases that underwent wide local excision had better local recurrence and OS rates. This may be attributed to the fact that the need for a more aggressive surgery to obtain a NED status reflects a less favorable response to preoperative treatment and more aggressive tumor biology. 

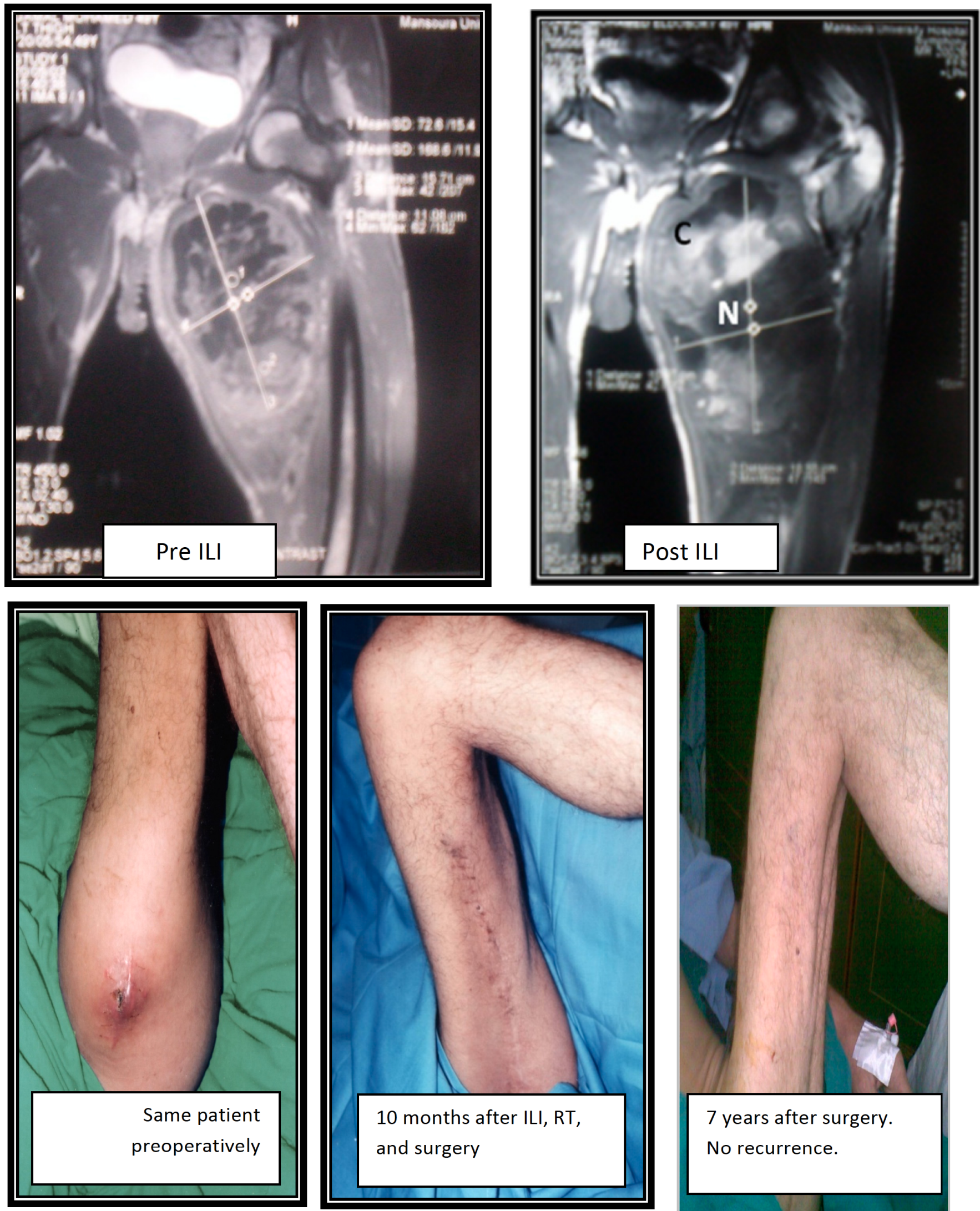

Fig. (5). MRI of Pleomorphic Liposarcoma in the posteromedial aspect of left thigh showed a minimal tumor volume response (18\%). Post treatment film show increased extent of necrosis $(\mathrm{N})$ and cystic degeneration $(\mathrm{C})$. Below are photos of the same patient at presentation, 10 months, and 7 years after ILI, External irradiation and surgery. 
Limitations of this retrospective study include difficulties for collecting data. Twenty four percent of Group II patients were coming from outside the referring population of our University Hospital. The present study is a retrospective review of ASTS cases treated by ILI and NACT in our center. It is very heterogeneous when regarding different histopathological diagnoses, different TNM and different stages together in the same series. However it is homogeneous and different from other series in that there are almost only large size $(>5 \mathrm{~cm})$ and high stages (IIa-IV). There is a selection bias and the authors acknowledge this. This is not a randomized trial and comparison between groups could never be that perfect although the differences between both groups were statistically insignificant.

\section{CONCLUSION}

In 2006, the authors inaugurated ILI as a simple method that provides a novel therapy in order to obtain local control and avoid amputation in cases of limb threatening soft tissue sarcoma. In this study, assessing the long term outcome of ILI in the treatment of advanced-stage STS, a 12-year experience has been analyzed and presented. ILI cases experienced not only better tumor response rates but also significantly better local recurrence and overall survival rates than a comparable group of cases treated by preoperative systemic chemotherapy. Tumor response to ILI (and not systemic NACT) was associated with a better disease free and overall survival.

\section{ACKNOWLEDGEMENT}

We are grateful to our colleagues at the surgery department of Mansoura University Cancer Center. This work was supported by internal fund from Mansoura University Oncology Center.

\section{CONFLICT OF INTEREST}

None declared.

\section{REFERENCES}

[1] Bramwell VH. Controversies in surgical oncology: routine anthracycline based adjuvant chemotherapy for stage III extremity soft tissue sarcoma. Ann Surg Oncol 2006; 14(4): 1254-6.

[2] Zagars GK, Ballo MT, Pisters PW, et al. Surgical margins and reresection in the management of patients with soft tissue sarcoma using conservative surgery and radiation therapy. Cancer 2003; 97 : 2544-53.

[3] Bechter OE. Systemic therapy of soft tissue sarcoma - "nothing new under the sun?" ASCO 2009. Memo 2009; 2: 208-10.

[4] Oda Y, Tamiya S, Oshiro $\mathrm{Y}$, et al. Re assessment and clinicopathological prognostic factors of malignant fibrous histocytoma of soft tissue parts. Pathol Int 2002; 52: 595.

[5] Pisters PW, Ballo MT, Patel SR. Preoperative chemoradiation treatment strategies for localized sarcoma. Ann Surg Oncol 2002; 9: $535-42$
[6] Thompson JF, Kam PC, Waugh RC, et al. Isolated limb infusion with cytotoxic agents: a simple alternative to isolated limb perfusion. Semin Surg Oncol 1988; 14: 238-47.

[7] Hegazy MA, Kotb S, Sakr H, et al. Preoperative isolated limb infusion of doxorubicin and external irradiation for limb threatening soft tissue sarcomas. Ann Surg Oncol 2007; 14: 56876.

[8] Wieberdink J, Benchkhuysen C, Braat RP, et al. dosimetry in isolation perfusion of the limbs by assessment of the perfused tissue volume and grading of toxic tissue reactions. Eur J Cancer Clin Oncol 1982; 18: 905-10.

[9] Krementz ET, Carter RD, Sutherland CM, et al. Regional chemotherapy for melanoma. A 35-year experience. Ann Surg 1994; 220: 520-34

[10] Lindner P, Doubrovsky A, Kam PC, et al. Prognostic factors after isolated limb infusion with cytotoxic agents for melanoma. Ann Surg Oncol 2002; 9: 127-36.

[11] Moncrieff M, Kroon HM, Kam PC, et al. Results of isolated limb infusion in advanced soft tissue sarcoma of the extremity. Ann Surg Oncol 2008; 15: 2749-56.

[12] Cherix S, Speiser M, Matter M, et al. Isolated limb perfusion with tumor necrosis factor and melphalan for non-resectable soft tissue sarcomas: long-term results on efficacy and limb salvage in a selected group of patients. J Surg Oncol 2008; 98: 148-55.

[13] Noorda EM, Vrouenraets BC, Nieweg OE, et al. Isolated limb perfusion with tumor necrosis factor-alpha and melphalan for patients with unresectable soft tissue sarcoma of the extremities. Cancer 2003; 98: 1483-90.

[14] Meric F, Hess KR, Varma DG, et al. Radiographic response to neoadjuvant chemotherapy is a predictor of local control. Cancer 2002; 95: 1120-6.

[15] Menendez LR, Ahlmann ER, Savage K, et al. Tumor necrosis has no prognostic value in neoadjuvant chemotherapy for soft tissue sarcoma. CORR 2006; 455: 219-24.

[16] Eilber FC, RosenG, Eckardt J, et al. Treatment-induced pathologic necrosis: a predictor of local recurrence and survival in patients receiving neoadjuvant therapy for high-grade extremity soft tissue sarcomas. J Clin Onc 2001; 19: 3203-9.

[17] Halperna L, Gilbertb J, Ginger E, et al. Chemotherapy for soft tissue sarcoma. Curr Opin Orthopaed 2007; 18: 604-10.

[18] Gortzak E, Azzarelli A, Buesa J, et al. A randomised phase II study on neo-adjuvant chemotherapy for 'high-risk' adult softtissue sarcoma. Eur J Cancer 2001; 37: 1096-103.

[19] Van Glabbeke M, Van Oosterom AT, Oosterhuis JW, et al. Prognostic factors for the outcome of chemotherapy in advanced soft tissue sarcoma: an analysis of 2,185 patients treated with anthracycline-containing first-line regimens - a European Organization of Research and Treatment of Cancer Soft Tissue and Bone Sarcoma Group. J Clin Oncol 1999; 17: 150-67.

[20] Collin CF, Friedrich C, Godbold J, et al. Prognostic factors for local recurrence and survival in patients with localized extremity soft tissue sarcoma. Semin Surg Oncol 1988; 4: 30-7.

[21] Sanki A, Kam P, Tghompson J. long term results of hyperthermic, isolated limb perfusion for melanoma: a reflection of tumor biology. Ann Surg 2007; 245: 591-6.

[22] Issels RD, Abdel-Rahman S, Wendtner M, et al. Neoadjuvant chemotherapy combined with regional hyperthermia (RHT) for locally advanced primary or recurrent high-risk adult soft-tissue sarcomas (STS) of adults: long-term results of a phase II study. Eur J Cancer 2001; 37: 1599-608.

This is an open access article licensed under the terms of the Creative Commons Attribution Non-Commercial License (http://creativecommons.org/licenses/by-nc/ $3.0 /$ ) which permits unrestricted, non-commercial use, distribution and reproduction in any medium, provided the work is properly cited. 